\title{
GuaLou GuiZhi decoction inhibits LPS-induced microglial cell motility through the MAPK signaling pathway
}

\author{
HAIXIA HU ${ }^{1,2,4}$, ZUANFANG LI ${ }^{1,4}$, XIAOQIN ZHU ${ }^{2,4}$, RUHUI LIN ${ }^{3,4}$, \\ JUN PENG $^{3,4}$, JING TAO ${ }^{1,2}$ and LIDIAN CHEN ${ }^{1,2,3}$ \\ ${ }^{1}$ Key Laboratory of TCM Rehabilitation of State Administration of Traditional Chinese Medicine, \\ ${ }^{2}$ Fujian Rehabilitation Technology Engineering Center, ${ }^{3}$ MOE Key Laboratory of Traditional Chinese Medicine \\ on Osteology and Traumatology and Exercise Rehabilitation, ${ }^{4}$ Academy of Integrative Medicine, \\ Fujian University of Traditional Chinese Medicine, Fuzhou, Fujian, P.R. China
}

Received June 18, 2013; Accepted September 13, 2013

DOI: $10.3892 / \mathrm{ijmm} .2013 .1522$

\begin{abstract}
Microglial activation plays an important role in neroinflammation following ischemic stroke. Activated microglial cells can then migrate to the site of injury to proliferate and release substances which induce secondary brain damage. It has been shown that microglial migration is associated with the activation of the mitogen-activated protein kinase (MAPK) signaling pathways. The Chinese formula, GuaLou GuiZhi decoction (GLGZD), has long been administered in clinical practice for the treatment of post-stroke disabilities, such as muscular spasticity. In a previous study, we demonstrated that the anti-inflammtory effects of GLGZD were mediated by the TLR4/NF- $\mathrm{B}$ pathway in lipopolysaccharide (LPS)-stimulated microglial cells. Therefore, in this study, we evaluated the role of GLGZD in microglial migration by performing scratch wound assays and migration assays. We wished to elucidate the cellular and molecular mechanisms elicited by this TCM formula in microglial-induced inflammation by evaluating the release and expression of chemotactic cytokines [monocyte chemoattractant protein-1 (MCP-1), macrophage inflammatory protein-1 $\alpha$ (MIP-1 $\alpha$ ) and interleukin (IL)-8] by ELISA and quantitative PCR. Our results revealed that the migration of microglial cells was enhanced in the presence of LPS
\end{abstract}

Correspondence to: Dr Jing Tao or Dr Lidian Chen, Fujian Rehabilitation Technology Engineering Center, Fujian University of Traditional Chinese Medicine, 1 Huatuo Road, Minhou Shangjie, Fuzhou, Fujian 350108, P.R. China

E-mail: taojing01@yahoo.com.cn

E-mail: cld@fjtcm.edu.cn

Abbreviations: GLGZD, Gua Lou Gui Zhi decoction; CNS, central nervous system; LPS, lipopolysaccharide; MCP-1, monocy te chemoattractant protein-1; MIP-1 $\alpha$, macrophage inflammatory protein-1 $\alpha$; IL-8, interleukin-8; MAPKs, mitogen-activated protein kinases; JNK, c-Jun N-terminal protein kinase; ERK1/2, extracellular signal-regulated kinase $1 / 2$

Key words: Gua Lou Gui Zhi decoction, stroke, microglia, inflammation, migration
(100 ng/ml); however, GLGZD (100 $\mu \mathrm{g} / \mathrm{ml})$ significantly inhibited cell motility and the production of chemokines through the inhibition of the activation of the p38 and c-Jun N-terminal protein kinase (JNK) signaling pathway. We demonstrate the potential of GLGZD in the modulation of microglial motility by investigating the effects of GLGZD on microglial migration induced by LPS. Taken together, our data suggest that GLGZD per se cannot trigger microglial motility, whereas GLGZD impedes LPS-induced microglial migration through the activation of the MAPK signaling pathway. These results provide further evidence of the anti-inflammatory effects of GLGZD and its potential for use in the treatment of ischemic stroke.

\section{Introduction}

Stroke is a leading cause of mortality and disability worldwide and $70-80 \%$ of all cases are ischemic stroke (1). Post-ischemic inflammation is an essential step in the progression of ischemic stroke (2). Microglial cells constitute the resident immune cell population of the mammalian central nervous system (CNS), which play a pivotal role in neuroinflammation by avidly surveying the brain parenchyma $(3,4)$. In this sense, microglial cells become activated and migrate to the injury site in order to fully develop a concerted immune response, involving the release of both trophic and pro-inflammatory factors, such as tumor necrosis factor- $\alpha$ (TNF- $\alpha$ ), interleukin (IL)-6 and IL-1 $\beta$ and nitric oxide (NO), which are believed to lead to severe neuronal death and brain injury $(5,6)$. Chemokines [monocyte chemoattractant protein-1 (MCP-1), IL-8 and macrophage inflammatory protein-1 $\alpha$ (MIP-1 $\alpha)$ ] produced by microglial cells are also important enhancers of post-ischemic inflammation $(7,8)$. Gua Lou Gui Zhi decoction (GLGZD) is a classical traditional Chinese formula that was first prescribed in the Eastern Han dynasty, around 210 AD and it consists of a combination of six herbs, including Trichosanthis Radix, Ramulus Cinnamomi, Paeonia lactiflora, Glycyrrhiza, Zingiber officinale Roscoe and Fructus Jujubae. It has long been used in China in clinical practice for the treatment of post-stroke disabilities, such as muscular spasticity (9). We have previously demonstrated that GLGZD exerts inhibitory effects on LPS-induced inflammatory events in microglial cells (9). The anti-inflammatory effects of 
GLGZD prompted us to examine the effects of GLGZD on microglial migration. The signal transduction pathways in $\mathrm{BV}-2$ microglial cells induced by lipopolysaccharide (LPS), a bacterial endotoxin widely used for studying experimental inflammation, may eventually lead to the phosphorylation of the mitogenactivated protein kinase (MAPK) signaling pathways, resulting in the production of inflammatory mediators, including chemotactic cytokines. In the present study, we attempted to evaluate the effects of GLGZD on LPS-induced microglial motility and the regulation of chemotactic cytokines by GLGZD in activated BV-2 microglial cells. We demonstrate that GLGZD ultimately protects neurons from secondary damage following ischemic stroke.

\section{Materials and methods}

Materials and reagents. LPS (from Escherichia coli 055:B5) and 3-[4,5-dimethyl-2-thiazolyl]-2,5-diphenyltetrazoliumbromide (MTT) were obtained from Sigma-Aldrich (St. Louis, MO, USA). FBS, DMEM, penicillin, streptomycin, $0.05 \%$ (w/v) trypsin/EDTA and phosphate-buffered saline (PBS) were obtained from Gibco-BRL (Gaithersburg, MD, USA). The cytokine (MCP-1, MIP-1 $\alpha$ and IL-8) ELISA kits were purchased from R\&D Systems (Minneapolis, MN, USA). The real-time PCR kit was purchased from (Takara, Shiga, Japan). The antibodies used for western blot analysis in this study included: mouse p38 monoclonal antibody (mAb), phospho-p38 $\mathrm{mAb}$, c-Jun N-terminal protein kinase (JNK) mAb, phosphoJNK mAb, extracellular signal-regulated kinase 1/2 (ERK1/2) $\mathrm{mAb}$, phospho-ERK1/2 mAb and $\beta$-actin mAb (Santa Cruz Biotechnology, Inc., Santa Cruz, CA, USA) and horseradish peroxidase (HRP)-conjugated secondary antibodies were from Cell Signaling Technology, Inc. (Beverly, MA, USA).

Preparation of water extract of GLGZD. Medicinal plants were supplied by the Guo Yi Tang Chinese Herbal medicine store (Fuzhou, China). The preparation of the GLGZD water extract was performed as described in our previous study (9). The stock and working concentrations of GLGZD were prepared by dissolving the extract in culture medium to a concentration of $50 \mathrm{mg} / \mathrm{ml}$ and $100 \mu \mathrm{g} / \mathrm{ml}$.

Cell line culture. BV-2 murine microglial cells were maintained in DMEM supplemented with $10 \%$ fetal bovine serum, $100 \mathrm{U} / \mathrm{ml}$ penicillin and $100 \mu \mathrm{g} / \mathrm{ml}$ streptomycin at $37^{\circ} \mathrm{C}$ in a humidified incubator under $5 \% \mathrm{CO}_{2}$. Cells were plated at a density of $1 \times 10^{5}$ cells per well in 96-well trays (MTT assays), $8 \times 10^{4}$ cells per well in 24-well trays (ELISA) or plated at a density of $5 \times 10^{5}$ cells per well in 6-well trays (for the remaining experiments). Cell treatments included the following incubation setup: serum-free medium for $4 \mathrm{~h}$ prior to cell treatment, LPS (100 ng/ml, Sigma) and/or GLGZD water extract $(100 \mu \mathrm{g} / \mathrm{ml})$ for $12 \mathrm{~h}$ (mRNA expression evaluation, cytokine release and migration analyses) or $24 \mathrm{~h}$ (cytotoxicity assays).

MTT assay. BV-2 microglial cell viability was assessed by MTT assay. MTT is actively catalyzed by mitochondrial succinate dehydrogenase to form dark blue formazan in live cells. The formation of formazan is therefore used as an indicator of cell viability. BV-2 cells cultured in 96-well plates were treated with
GLGZD at various concentrations and LPS for $24 \mathrm{~h}$, followed by incubation with MTT $(0.5 \mathrm{mg} / \mathrm{ml})$ for an additional $4 \mathrm{~h}$. The formazan formed in the cells was dissolved by dimethyl sulfoxide (DMSO; Sigma-Aldrich) and read at $570 \mathrm{~nm}$ using a microplate absorbance reader (EXL 808; BioTek Instruments, Bad Friedrichshall, Baden-Wurttemberg, Germany).

Scratch wound assay. The BV-2 cells were seeded in 6-well plates in monolayer until approximately $95 \%$ confluent prior to the migration assay. Four hours before performing the sratch wound assay, the medium was replaced with serum-free medium to ensure that no proliferation occurred during the experiments. The wound was made by a perpendicular scratch made with a pipette tip. The cells were stimulated with $100 \mathrm{ng} /$ $\mathrm{ml} \mathrm{LPS,} 100 \mu \mathrm{g} / \mathrm{ml}$ GLGZD or $100 \mathrm{ng} / \mathrm{ml} \mathrm{LPS}+100 \mu \mathrm{g} / \mathrm{ml}$ GLGZD for $12 \mathrm{~h}$. Following treatment, microphotographs of the cells that had migrated into the open wound were taken.

Transwell migration assay. In this study, the Costar Transwell System (8- $\mu \mathrm{m}$ pore size polycarbonate membrane, Costar, Cambridge, MA, USA) was used to evaluate vertical cell migration. BV-2 cells in $0.2 \mathrm{ml}$ serum-free medium were seeded in the upper well and $0.6 \mathrm{ml} 100 \mathrm{ng} / \mathrm{ml} \mathrm{LPS,} 100 \mu \mathrm{g} / \mathrm{ml}$ GLGZD, $100 \mathrm{ng} / \mathrm{ml}$ LPS + $100 \mu \mathrm{g} / \mathrm{ml}$ GLGZD, or DMEM as the negative control were added to the lower chamber. At the end of a $24-\mathrm{h}$ incubation period at $37^{\circ} \mathrm{C}$, the cells on the upper side of the filters were removed with cotton-tipped swabs and the filters were fixed with $4 \%$ paraformaldehyde in PBS for $30 \mathrm{~min}$ and stained with crystal violet in PBS for 15 min followed by obtaining representative images using a microscope. The migrated cells on the lower surface of the membrane were then solubilized in extraction buffer. Optical density (OD) values at $540 \mathrm{~nm}$ were determined using a microplate reader.

Enzyme-linked immunosorbent assay (ELISA). The BV-2 cells were plated in a 24-well cell culture plate and incubated with GLGZD water extract $(100 \mu \mathrm{g} / \mathrm{ml})$ in the presence or absence of LPS (100 ng/ml) for $12 \mathrm{~h}$. Following treatment with the stimuli, ELISA kits were used according to the manufacturer's instructions for mouse MCP-1, MIP-1 $\alpha$ and IL-8. The OD values were recorded at $450 \mathrm{~nm}$.

Quantitative polymerase chain reaction. For gene expression analysis, the medium was removed following incubation and the BV-2 cells were washed with PBS twice. Total RNA was isolated from the BV-2 murine microglial cell cultures using TRIzol reagent (Invitrogen Corp., Carlsbad, CA, USA). Precipitated RNA was resuspended in $20 \mu \mathrm{l}$ of diethylpyrocarbonate (DEPC)-treated water and quantified by measuring the OD using a NanoDrop 2000 spectrophotometer (Thermo Scientific, Waltham, MA,USA). cDNA was synthesized using avian myeloblastosis virus reverse transcriptase (Takara), according to the manufacturer's instructions. Quantitative PCR was performed in triplicate using an Applied Biosystems Prism 7500 Fast Sequence Detection System (Applied Biosystems, Foster City, CA, USA) with the SYBR-Green I quantitative PCR kit according to the instructions of the manufacturer (Takara). The sequences of the primers were as follows: MCP-1 forward, 5'-TCGGAACCAAATGAGATCAGAAC-3' 
and reverse, 5'-GAGGTGGTTGTGGAAAAGGTAGTG-3' (135 bp); MIP-1 $\alpha$ forward, 5'-TCGTTGACTATTTTG AAACCAGCAG-3' and reverse, 5'-TCAGGCATTCAGTT CCAGGTCA-3' (140 bp); IL-8 forward, 5'-ATGCTCCTGCT GGCTGTCCT-3' and reverse, 5'-TTGGGACTGCTATCAC TTCCTTTC-3' (178 bp); and $\beta$-actin forward, 5'-GAGAGGG AAATCGTGCGTGACA-3' and reverse, 5'- ACCCAAG AAGGAAGGCTGGAAA-3' (192 bp) (Takara). Levels of RNA expression were determined using the 7500 Fast System (Applied Biosystems). The results were normalized to $\beta$-actin expression and analyzed relative to their untreated counterparts. The relative quantification of the gene of interest was determined using the comparative CT method $\left(2^{-\Delta \Delta C t}\right)$.

Western blot analysis. The cells were washed twice with cold PBS and lysed in ice-cold radioimmunoprecipitation buffer containing protease inhibitors [phenylmethylsulfonyl fluoride (PMSF)]. The lysate was centrifuged for $10 \mathrm{~min}$ at $12,000 \mathrm{x} \mathrm{g}$ at $4^{\circ} \mathrm{C}$ and the supernatant collected. Total protein concentration was quantified using the bicinchoninic acid (BCA) protein assay kit and the samples were stored at $-80^{\circ} \mathrm{C}$. Subsequently, protein $(50 \mu \mathrm{g})$ samples was separated by SDS-PAGE on $10 \%$ gels and then transferred onto PVDF membranes (Millipore, Billerica, MA, USA). The membranes were blocked in Tris-buffer for $1 \mathrm{~h}$ at room temperature and then incubated overnight at $4^{\circ} \mathrm{C}$ with primary antibodies: p38 and phospho-p38 mAb, JNK and phospho-JNK mAb, ERK1/2 and phospho-ERK1/2 mAb and $\beta$-actin $\mathrm{mAb}(1: 1,000)$. After rinsing with TBS-T, membranes were incubated for $1 \mathrm{~h}$ at room temperature with horseradish peroxidase-conjugated secondary antibodies: anti-goat, anti-rabbit or anti-mouse IgG $(1: 1,000)$. Protein immunoreactive bands were developed using the enhanced chemiluminescence (ECL)-Plus kit (RPN2132; GE Healthcare, Pittsburgh, PA, USA) for 1 min and chemiluminescent bands were detected on Kodak autoradiography film in a dark room, and their densities were measured using ImageJ software.

Data analysis. Data are presented as the means \pm standard error of the mean (SEM). Statistical analysis was performed using SPSS 15.0 software. One-way analysis was used for a comparison among the experimental conditions and a $\mathrm{P}$-value $<0.05$ was considered to indicate a statistically significant difference.

\section{Results}

GLGZD does not affect $B V-2$ cell viability. The cytotoxicity of GLGZD was evaluated in the presence or absence of LPS by MTT assay. GLGZD at a concentration of $100 \mu \mathrm{g} / \mathrm{ml} \mathrm{did}$ not significantly affect the viability of the BV-2 cells when the cells were incubated with or without LPS $(100 \mathrm{ng} / \mathrm{ml})$ for $24 \mathrm{~h}$ (Fig. 1). Hence, GLGZD at $100 \mu \mathrm{g} / \mathrm{ml}$ alone and co-treatment with LPS exerted no cytotoxic effects on the BV-2 microglial cells compared with the control group.

GLGZD exerts an inhibitory effect on LPS-induced microglial migration. To assess the effect of GLGZD on microglial motility, we performed a scratch wound assay and Transwell migration assay. As shown in Fig. 2A, representative micro-

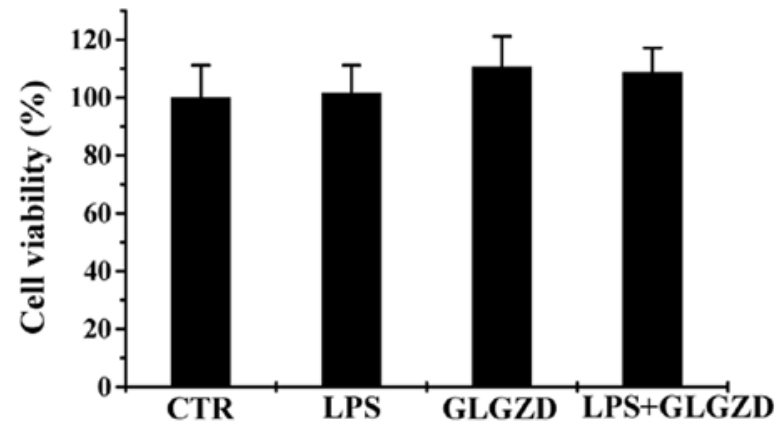

Figure 1. Effects of GuaLou GuiZhi decoction (GLGZD) on the viability of BV-2 microglial cells exposed to lipopolysaccharide (LPS). BV-2 cells were cultured for $4 \mathrm{~h}$ in serum-free medium followed by treatment with LPS alone or co-incubation with GLGZD for an additional $24 \mathrm{~h}$. Cell viability was determined by MTT assay and data are expressed as the means \pm SD of three independent experiments.

scopic images clearly illustrate that the LPS-activated BV-2 cells exhibited an enhanced migratory potential compared with the control; however, we observed that $100 \mu \mathrm{g} / \mathrm{ml} \mathrm{GLGZD}$ significantly reduced cell motility in the co-culture with LPS and GLGZD alone had no effect on microglial migration. Similar to the scratch assays, the Transwell migration assay over a period of $24 \mathrm{~h}$ indicated that the migratory capacity of the BV-2 cells was not altered by GLGZD alone; however, co-treatment with LPS decreased the number of migrating cells (Fig. 2B). The statistical analysis of three independent experiments revealed that GLGZD significantly reduced the number of migrating cells in the presence of LPS in the culture medium (Fig. 2C). In both assays, GLGZD significantly attenuated microglial migration.

GLGZD attenuates the LPS-stimulated release of chemotactic cytokines at the transcriptional and translational levels in $B V-2$ cells. Activated microglial cells produce chemokines, inducing immune cells to migrate to the injured brain site following ischemic stroke. To explore the effects of GLGZD on the production of several chemokines associated with microglial motility, following incubation with GLGZD in the presence or absence of LPS, cell supernatant was collected to examine the release of chemokines in BV-2 cells. As indicated in Fig. 3A-C, the incubation of BV-2 cells with LPS $(100 \mathrm{ng} /$ $\mathrm{ml}$ ) for $12 \mathrm{~h}$ resulted in a significant increase in MCP-1, MIP-1 $\alpha$ and IL- 8 production. The concentrations of these chemokines were not significantly affected by GLGZD $(100 \mu \mathrm{g} / \mathrm{ml})$ alone (levels were similar to the control). However, the induction of these chemokines by LPS was reduced significantly by co-treatment with GLGZD. In parallel to the protein secretion levels, the LPS-induced upregulation of MCP-1, MIP-1 $1 \alpha$ and IL- 8 mRNA was attenuated by GLGZD $(100 \mu \mathrm{g} / \mathrm{ml})$, as measured at $12 \mathrm{~h}$ (Fig. 3D).

GLGZD supresses the LPS-induced phosphorylation of MAPK in $B V-2$ cells. It is well recognized that the LPS-mediated microglial migration is dependent on the phosphorylation of MAPK. The members of MAPK are p38, JNK and ERK1/2, which may be involved in the inhibitory effects of GLGZD on BV-2 microglial activation. The inhibitory effects of GLGZD 
A

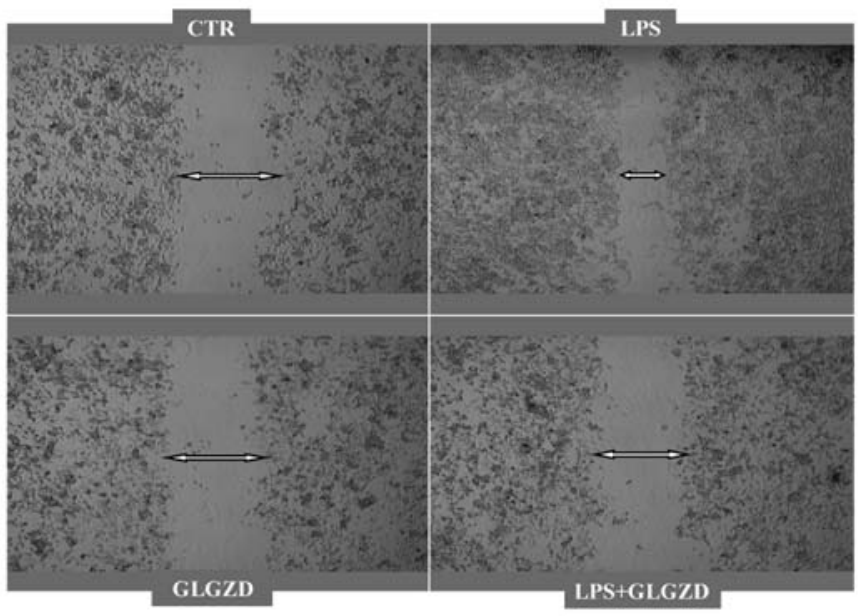

B

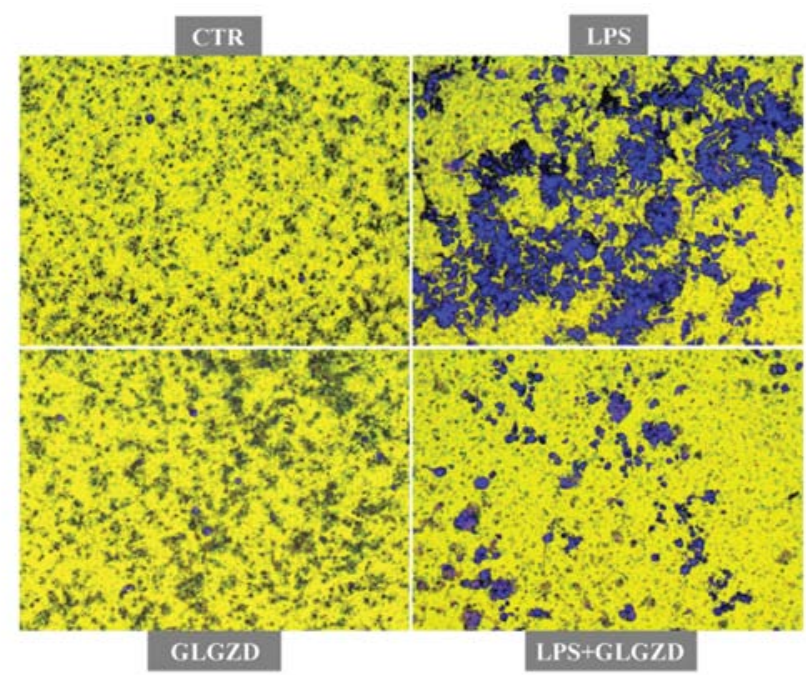

C

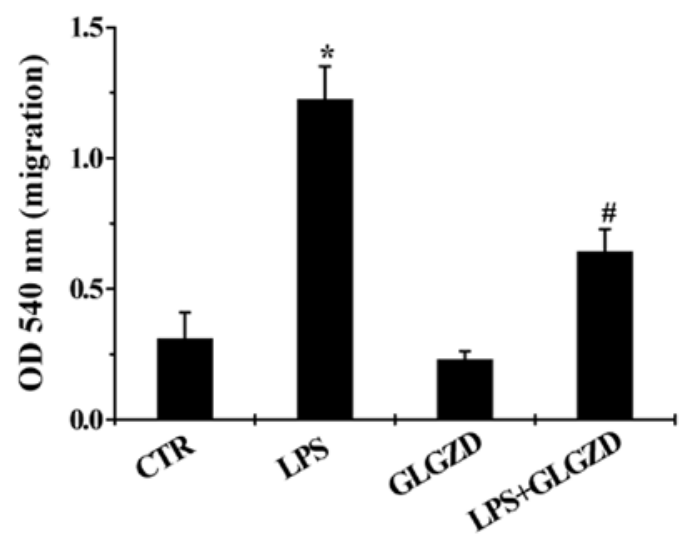

Figure 2. GuaLou GuiZhi decoction (GLGZD) supresses lipopolysaccharide (LPS)-induced microglial motility. (A) GLGZD inhibits the migratory potential of LPS-induced BV-2 cells, as shown by scratch wound assays. Micrographs from one representative experiment out of three independent experiments are shown. (B and C) Representative photomicrographs of crystal violet-stained BV-2 cells as per experimental condition were acquired. Transwell chamber migration assay indicated the inhibitory effects of GLGZD on the migration potential of LPS-induced BV-2 microglial cells. The ability of BV-2 cells to migrate through the filters was significantly suppressed by GLGZD, when compared with the LPS-treated cells. Absorbance correlating with the number of invaded cells was read at $540 \mathrm{~nm}$ (means $\pm \mathrm{SD} ; \mathrm{n}=3 ;{ }^{*} \mathrm{P}<0.05$ compared with untreated cells, ${ }^{\prime} \mathrm{P}<0.05$ compared with LPS-treated cells).
A

B
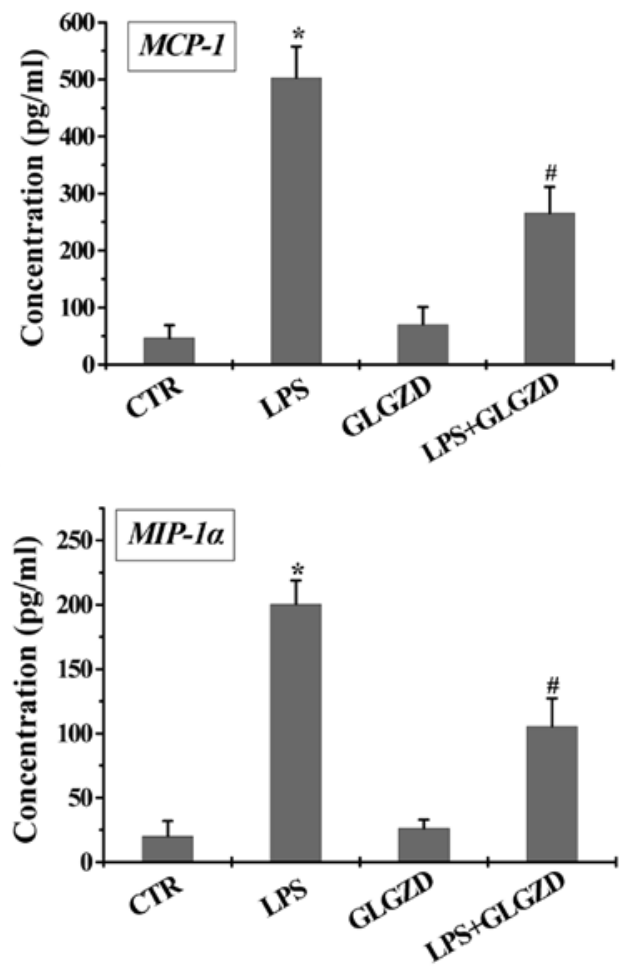

C

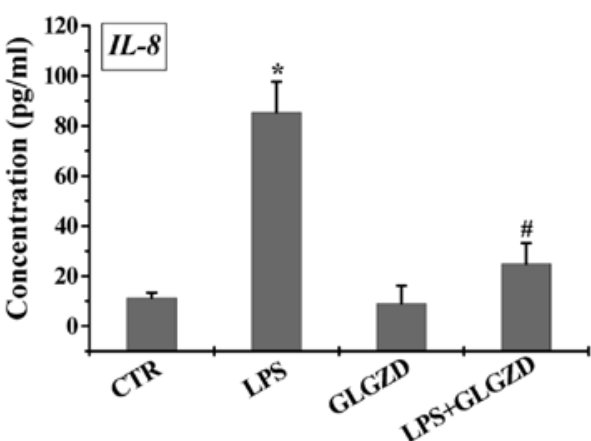

D

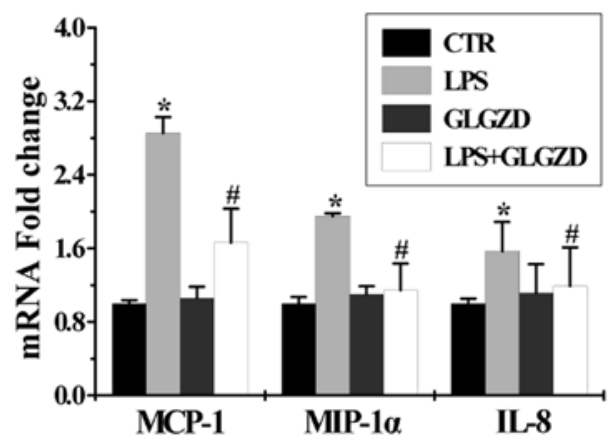

Figure 3. GuaLou GuiZhi decoction (GLGZD) suppresses the production and expression of chemotactic cytokines in lipopolysaccharide (LPS)-induced microglial cells. (A-C) BV-2 microglial cells were treated with LPS and/or GLGZD for the indicated periods of time. Culture supernatants were analyzed for monocyte chemoattractant protein-1 (MCP-1), MIP-1 $\alpha$, macrophage inflammatory protein-1 $\alpha$ (MIP-1 $\alpha$ ) and interleukin (IL)- 8 content by ELISA. (D) qRT-PCR validation of MCP-1, MIP-1 $\alpha$ and IL- 8 transcripts in BV-2 microglial cells stimulated with LPS and/or GLGZD for $12 \mathrm{~h}$. The expression was normalized to the control gene and mRNA levels are expressed as the fold induction relative to the control cells. Results are calculated from three independent experiments performed in triplicate measurements. " $\mathrm{P}<0.05 \mathrm{com}$ pared with untreated cells, ${ }^{\#} \mathrm{P}<0.05$ compared with LPS-treated cells. 
A

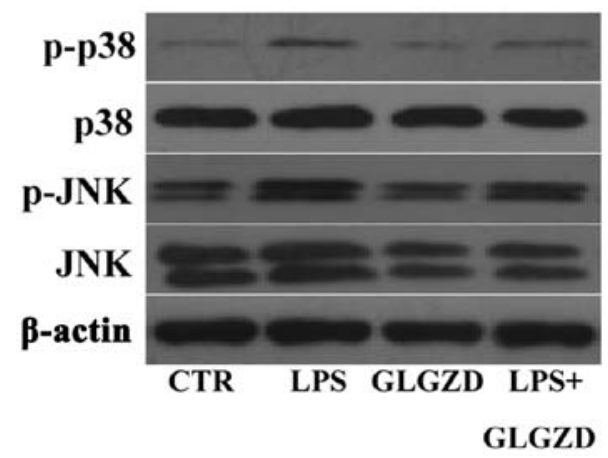

B

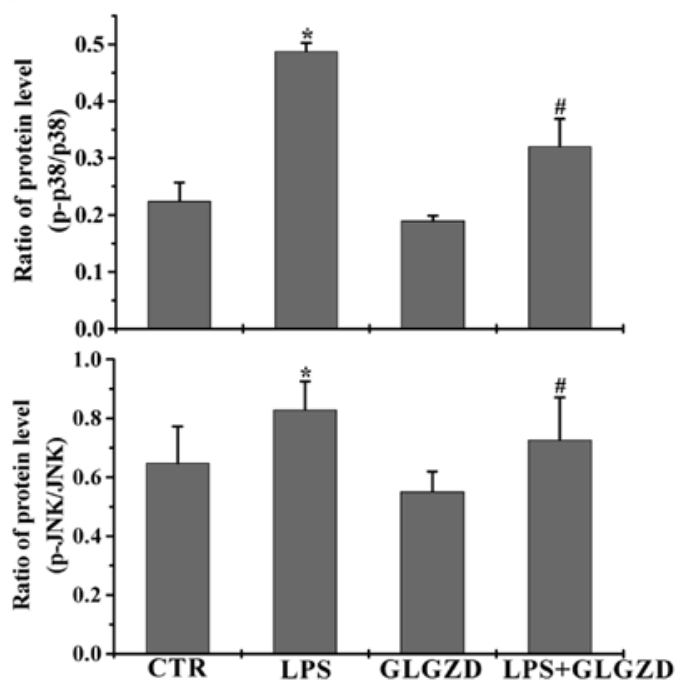

Figure 4. GuaLou GuiZhi decoction (GLGZD) inhibits the phosphorylation of MAPK in activated microglial cells. (A) A representative western blot showing the expression of phospho-p38 MAPK and JNK in lipopolysaccharide (LPS) and/or GLGZD-treated BV-2 cells. A representative experiment out of three is shown. (B) The relative protein band intensities were quantified by densitometric analyses and normalized to $\beta$-actin, total p38 MAPK and JNK. Data are shown as the means \pm SD of data from three independent experiments. ${ }^{*} \mathrm{P}<0.05$ compared with untreated cells, ${ }^{\text {}} \mathrm{P}<0.05$ compared with LPS-treated cells.

on cell motility are very likely mediated by the MAPK signaling pathway. To futher delineate the mechanisms of action of GLGZD in LPS-stimulated microglial migration, we evaluated the phosphorylation of MAPK in BV-2 cells. The phosphorylation of p38 and JNK induced by LPS $(100 \mathrm{ng} / \mathrm{ml})$ and/or GLGZD $(100 \mu \mathrm{g} / \mathrm{ml})$ was observed. The level of phospho-p38 and phospho-JNK significantly increased $1 \mathrm{~h}$ after the challenge with LPS and GLGZD significantly reduced the phosphorylation of p38 and JNK MAPK in the LPS-challenged BV-2 cells (Fig. 4), but did not have any effect on the phosphorylation of ERK1/2 (data not shown). These results suggested that JNK and p38 MAPK signaling was required for GLGZD to suppress the induction of chemokines in microglial cells.

\section{Discussion}

Microglial cells are the intrinsic immune cells of the brain and the main effectors of inflammatory response following cerebral ischemia (10), playing important roles in the physiological and pathological conditions of the CNS. Following injury to the
$\mathrm{CNS}$, microglia are rapidly activated, triggering inflammatory reactions at the sites of injury $(11,12)$. The migration of microglial cells is a hallmark of pro-inflammatory and chronic activation during the early phases of neurodegeneration. Chemokines released from microglial cells are key components required for cell movement. The overactivation of microglia, however, can be highly detrimental to neuronal cells, due to the release of several pro-inflammatory factors and chemotactic cytokines. Accordingly, the inhibition of microglial activation and the production of chemotactic cytokines may be beneficial in reversing microglial-mediated neurodegeneration.

The recognition that cerebral stroke is a manifestation of chronic progressive inflammation has had great influence on the development of therapeutic and preventative strategies $(13,14)$ and as such, the Chinese formula may be a promising therapeutic strategy for ameliorating strokeinduced inflammatory pathological events. GLGZD has been reported to be an effective treatment for stroke in clinical practice and in animal experiments (15-17). In our previous study in vitro, we demonstrated that GLGZD water extract exerts anti-inflammatory effects in LPS-induced microglial cells mediated by the nuclear factor $-\kappa \mathrm{B}(\mathrm{NF}-\kappa \mathrm{B})$ sinaling pathway (9); however, the precise molecular mechanisms responsible for its neuroprotective effects have not yet been elucidated. In this regard, we hypothesized that GLGZD may affect the motility of activated microglia. In the present study, we used LPS, a bacterial endotoxin used to experimentally study induced inflammation in vitro $(18,19)$, to promote the migration of microglial cells, thus creating a cell model of LPS-induced microglial activation. LPS activates NF- $\kappa \mathrm{B}$ and MAPK, which are classified into at least three components: ERK1/2, JNK and p38 MAPK (20). Certain studies have demonstrated that LPS-induced microglial migration requires p38 phosphorylation (21) and that the PI3K/Akt pathway correlates with microglial migration $(22,23)$. Therefore, in this study, we aimed to elucidate the mechanisms responsible for the effects of GLGZD on LPS-induced microglial migration by evaluating the role of the MAPK signaling pathways.

We demonstrated that LPS (100 ng/ml) and GLGZD (100 $\mu \mathrm{g} / \mathrm{ml})$ had no effect on microglial cell viability; thus, we selected the concentration of $100 \mu \mathrm{g} / \mathrm{ml}$ GLGZD for the remaining experiments. Our data demonstrated that GLGZD significantly inhibited microglial motility in LPS-activated BV-2 cells, as shown by scratch wound healing assays and Transwell migration assays. To further assess the effects of GLGZD on LPS-induced microglial migration, we examined the expression levels of several chemotactic mediators in BV-2 cells. LPS stimulated microglial cells to secrete representative chemokines, including MCP-1, MIP-1 $\alpha$ and IL-8; however, GLGZD markedly suppressed the LPS-induced production and expression of such cytokines (assessed by ELISA and quantitative RT-PCR). In addition, we sought to examine the modulatory effects of GLGZD on the MAPK singnaling pathway associated with the proliferation, survival and migration of microglial cells following ischemic stroke. We found that within $1 \mathrm{~h}$ after the stimulation of BV-2 cells with LPS, the phosphorylation of JNK and p38 MAPK was significantly enhanced but was considerably reduced in the cells co-cultured with LPS and GLGZD, indicating the involvement of JNK and p38 MAPK in the inhibitory effects of GLGZD on LPS-induced cell motility. 
Based on previous reports and our findings, GLGZD appears to be effective as an anti-inflammatory agent and exerts potent protective effects on the activation of microglia in multiple ways. Collectively, these findings further expand our current understanding of the regulatory mechanisms of GLGZD under inflammatory conditions following stroke in vitro, and provide a new range of therapeutic applications for GLGZD as an effective inflammatory modulator for the treatment of ischemic stroke.

\section{Acknowledgements}

This study was sponsored by grants from the Guidance Project of the Fujian Provincial Department of Science and Technology (no. 2012D012), the Key Project of the Department of Health of Fujian Province (no. zlckf01) and the Key Project of the Fujian Provincial Department of Science and Technology (no. 2012Y0041).

\section{References}

1. Shichita T, Ago T, Kamouchi M, Kitazono T, Yoshimura A and Ooboshi $\mathrm{H}$ : Novel therapeutic strategies targeting innate immune responses and early inflammation after stroke. J Neurochem 123 (Suppl 2): 29-38, 2012.

2. Shichita T, Sakaguchi R, Suzuki M and Yoshimura A: Postischemic inflammation in the brain. Front Immunol 3: 132, 2012.

3. Garden GA and Möller T: Microglia biology in health and disease. J Neuroimmune Pharmacol 1: 127-137, 2006.

4. Block ML, Zecca L and Hong JS: Microglia-mediated neurotoxicity: uncovering the molecular mechanisms. Nat Rev Neurosci 8: 57-69, 2007.

5. Nam KN, Koketsu M and Lee EH: 5-Chloroacetyl-2-amino-1,3selenazoles attenuate microglial inflammatory responses through NF-kappaB inhibition. Eur J Pharmacol 589: 53-57, 2008.

6. Choi Y, Lee MK, Lim SY, Sung SH and Kim YC: Inhibition of inducible NO synthase, cyclooxygenase-2 and interleukin-1beta by torilin is mediated by mitogen-activated protein kinases in microglial BV2 cells. Br J Pharmacol 156: 933-940, 2009.

7. Choi IY, Lee JC, Ju C, Hwang S, Cho GS, Lee HW, Choi WJ, Jeong LS and Kim WK: A3 adenosine receptor agonist reduces brain ischemic injury and inhibits inflammatory cell migration in rats. Am J Pathol 179: 2042-2052, 2011.

8. McLarnon JG: Microglial chemotactic signaling factors in Alzheimer's disease. Am J Neurodegener Dis 1: 199-204, 2012.
9. Hu H, Li Z, Zhu X, Lin R, Lin J, Peng J, Tao J and Chen L: Gua Lou Gui Zhi decoction suppresses LPS-induced activation of the TLR4/NF- $\kappa$ B pathway in BV-2 murine microglial cells. Int J Mol Med 31: 1327-1332, 2013.

10. Dirnagl U, Iadecola C and Moskowitz MA: Pathobiology of ischaemic stroke: an integrated view. Trends Neurosci 22: 391-397, 1999.

11. Bethea JR and Dietrich WD: Targeting the host inflammatory response in traumatic spinal cord injury. Curr Opin Neurol 15: $355-360,2002$.

12. Bareyre FM and Schwab ME: Inflammation, degeneration and regeneration in the injured spinal cord: insights from DNA microarrays. Trends Neurosci 26: 555-563, 2003.

13. Valtysson J, Hillered L, Andiné P, Hagberg H and Persson L: Neuropathological endpoints in experimental stroke pharmacotherapy: the importance of both early and late evaluation. Acta Neurochir (Wien) 129: 58-63, 1994.

14. Amantea D, Nappi G, Bernardi G, Bagetta G and Corasaniti MT: Post-ischemic brain damage: pathophysiology and role of inflammatory mediators. FEBS J 276: 13-26, 2009.

15. Zhang L and Ai H: Effects of Gua Lou Gui Zhi decoction on c-fos and c-jun on epileptic rats. J Sichuan Tradit Chin Med 23: 21-22, 2005.

16. Yang C, Chen L and Tao J: New usage of a classical formula - Gua Lou Gui Zhi decoction. Liaoning J Tradit Chin Med 8: 166-167, 2012.

17. Huang J, Tao J, Xue X, Yang S, Han P, Lin Z, Xu W, Lin J, Peng J and Chen L: Gua Lou Gui Zhi decoction exerts neuroprotective effects on post-stroke spasticity via the modulation of glutamate levels and AMPA receptor expression. Int J Mol Med 31: 841-848, 2013.

18. Rivest S: Molecular insights on the cerebral innate immune system. Brain Behav Immun 17: 13-19, 2003.

19. Cohen J: The immunopathogenesis of sepsis. Nature 420: 885-891, 2002.

20. Ajmone-Cat MA, De Simone R, Nicolini A and Minghetti L: Effects of phosphatidylserine on p38 mitogen activated protein kinase, cyclic AMP responding element binding protein and nuclear factor-kappaB activation in resting and activated microglial cells. J Neurochem 84: 413-416, 2003.

21. Ferreira R, Santos T, Cortes L, Cochaud S, Agasse F, Silva AP, Xapelli S and Malva JO: Neuropeptide Y inhibits interleukin-1 beta-induced microglia motility. J Neurochem 120: 93-105, 2012.

22. Horvath RJ and DeLeo JA: Morphine enhances microglial migration through modulation of $\mathrm{P} 2 \mathrm{X} 4$ receptor signaling. J Neurosci 29: 998-1005, 2009.

23. Martin S, Vincent JP and Mazella J: Involvement of the neurotensin receptor-3 in the neurotensin-induced migration of human microglia. J Neurosci 23: 1198-1205, 2003. 